\title{
Body size and activity times mediate mammalian responses to climate change
}

\author{
CHRISTY M. MCCAIN and SARAH R. B. KING \\ Department of Ecology \& Evolutionary Biology and CU Museum of Natural History, University of Colorado, 265 UCB, Boulder, \\ CO 80309, USA
}

\begin{abstract}
Model predictions of extinction risks from anthropogenic climate change are dire, but still overly simplistic. To reliably predict at-risk species we need to know which species are currently responding, which are not, and what traits are mediating the responses. For mammals, we have yet to identify overarching physiological, behavioral, or biogeographic traits determining species' responses to climate change, but they must exist. To date, 73 mammal species in North America and eight additional species worldwide have been assessed for responses to climate change, including local extirpations, range contractions and shifts, decreased abundance, phenological shifts, morphological or genetic changes. Only $52 \%$ of those species have responded as expected, $7 \%$ responded opposite to expectations, and the remaining $41 \%$ have not responded. Which mammals are and are not responding to climate change is mediated predominantly by body size and activity times (phylogenetic multivariate logistic regressions, $P<0.0001$ ). Large mammals respond more, for example, an elk is 27 times more likely to respond to climate change than a shrew. Obligate diurnal and nocturnal mammals are more than twice as likely to respond as mammals with flexible activity times $(P<0.0001)$. Among the other traits examined, species with higher latitudinal and elevational ranges were more likely to respond to climate change in some analyses, whereas hibernation, heterothermy, burrowing, nesting, and study location did not influence responses. These results indicate that some mammal species can behaviorally escape climate change whereas others cannot, analogous to paleontology's climate sheltering hypothesis. Including body size and activity flexibility traits into future extinction risk forecasts should substantially improve their predictive utility for conservation and management.
\end{abstract}

Keywords: behavior, elevation, latitude, mammal, microclimate, physiology, thermal niches

Received 22 September 2013 and accepted 4 December 2013

\section{Introduction}

In the past 50 years, temperatures have increased, precipitation regimes have changed, arctic ice has shrunk, and extreme weather events have increased in frequency due to anthropogenic modification of climate (Trenberth et al., 2007; USGCRP, 2009; Duffy \& Tebaldi, 2012). Various plants and animals have responded within individual populations and across their geographic and elevational ranges (Grabherr et al., 1994; Walther et al., 2002; Root et al., 2003; Parmesan, 2006; Lenoir et al., 2008; Chen et al., 2009; Myers et al., 2009). Most predictive modeling of mammals still assumes that all species will respond to climate change, will respond similarly albeit possibly at different rates, and directionally in step with temperature (e.g., Lawler et al., 2009; McCain \& Colwell, 2011). In contrast, ectotherm vertebrate modeling has incorporated physiological differences among species to make discriminating predictions about species responses to climate change (Buckley, 2008; Aragón et al., 2010; Huey et al., 2012).

Correspondence: Christy M. McCain, tel. + 1303735 1016; fax + 1 303492 4195, e-mail: christy.mccain@colorado.edu
Individual mammal studies (e.g., Moritz et al., 2008; Myers et al., 2009) demonstrate that some mammals have responded to climate change while others have not, even though all were predicted to respond similarly in climate envelope modeling. Despite the acknowledgement of the potential importance of species' traits in modifying responses to climate change (e.g., Parmesan, 2006; Buckley, 2008; Moritz et al., 2008; Angert et al., 2011; Huey et al., 2012), efforts to pinpoint such traits for endotherms so far are unsuccessful (Angert et al., 2011). Nonetheless, a better understanding of which species are responding and if there are consistent biological reasons for those responses is a conservation and management imperative. It is clear from analyses of mammalian fossil records that not all mammals responded similarly or directionally with paleo-temperature change (e.g., Lyons, 2003; Barnosky et al., 2004; Blois et al., 2010), but many physiological or behavioral traits cannot be analyzed in the fossil record due to lack of pertinent biological information or taphonomic biases. Three sets of general factors could influence whether particular species exhibit responses-(i) species' traits, particularly those that influence interactions 
with climate; (ii) locations, particularly areas that are experiencing greater climate change; and (iii) phylogeny, in that particular mammal clades may be responding more than others due to shared evolutionary history. Documentation of mammalian responses to recent climate change have accumulated rapidly in North America (147 populations of 73 species; Appendix S1), and eight species have been studied in other regions (e.g., Adamik \& Král, 2008; Lundy et al., 2010; Moyes et al., 2011; Appendix S2). These documented climate change responses, including local population extirpations, range contractions, range shifts, and directional change in abundance, phenology, body size, and genetic diversity (Appendix S1), offer an opportunity to test for species' traits, locality trends, and clade associations which may underlie the variability in responses to current climate change.

Many species' traits could influence climate change response from metabolism to food web interactions, but traits that directly impact the climate experienced by the individual may be most influential. Paleontologists have theorized that certain characteristics of mammals, including small body size, nocturnal behavior, and burrowing, may have allowed them to 'shelter' from the rapid climate change associated with the K-T extinction event in the Cenozoic in contrast to the much larger dinosaurs which perished (Robertson et al., 2004). Evidence linking mammal body size to differential extinction rates and large-scale range shifts during the K-T and Pleistocene extinction events lends some support to the climate sheltering theory (e.g., Lyons, 2003; Barnosky et al., 2004; Gingerich, 2006; Blois et al., 2010). In the current anthropogenic extinction crisis, large body size has been correlated with higher extinction risk status on the IUCN Red List, whereas other mammalian species' traits like burrowing and hibernating behaviors have been correlated with lower extinction risk status (e.g., Cardillo et al., 2005; Liow et al., 2009). Body size of terrestrial mammals spans orders of magnitude from $2 \mathrm{~g}$ to $1000 \mathrm{~kg}$, and the environments available to different sized mammals vary considerably. Small mammals can live within and under vegetation and soil which mediates the experienced temperature and humidity levels, whereas large mammals are necessarily above the vegetation and have fewer microclimate opportunities (e.g., Cardillo et al., 2005; Feldhamer et al., 2007). Alternatively, large mammals are more mobile and may be more able to track climate change and to detect isolated refuges that may be inaccessible to less mobile, small mammals (Angert et al., 2011; Schloss et al., 2012). When a mammal is active can also influence the temperature and humidity ranges it encounters. Particularly for species obligately active at certain times of the day, they must experience the abbreviated range of temperatures and humidity of that time interval. In contrast, species that are flexible in their activity times, for example nocturnal in the summer and diurnal in the winter, can select the range of temperatures and humidity in which they are active (e.g., Nowak, 1991; Feldhamer et al., 2007). Mammals that burrow under the soil or actively construct nests are able to live in a moderated microclimate not available to mammals without those traits (e.g., Kay, 1977; Bulova, 2002; Cardillo et al., 2005; Liow et al., 2009). Lastly, mammals capable of heterothermy, either seasonal (hibernation) or daily (torpor, estivation), can escape repeated extreme temperature and precipitation fluctuations that other mammals cannot (e.g., marmots vs. caribou in Alaska; e.g., Feldhamer et al., 2007; Liow et al., 2009). All of these traits influence the range of temperatures and humidity a mammal experiences in a particular location where some organisms have a wider array of microclimatic options and others have fewer. Those species that can broaden their microclimate choices and the range of temperatures and humidity experienced through modification of habitat choices, activity times, and behavioral temperature regulation may be less susceptible to climate change, while those species with a reduced range of options may have greater exposure to climate change and therefore be more sensitive and responsive.

Responses to climate change are predicted to be concentrated geographically, particularly at high latitudes and elevations, and at range edges (Grabherr et al., 1994; Walther et al., 2002; Parmesan \& Yohe, 2003; Root et al., 2003; Hickling et al., 2006). Thus, variation in response rate among mammals may be related to the severity of the climate change in the study location. For example, temperatures are increasing at a faster rate at high latitudes (Trenberth et al., 2007; USGCRP, 2009), and mammal populations at higher latitudes may respond more (e.g., Post \& Forchhammer, 2008; Gleason \& Rode, 2009). Species restricted to the highest latitudes and highest elevations are already near their climatic and geographic limits, and may also be responding at a higher rate (Grabherr et al., 1994; Parmesan \& Yohe, 2003; Chen et al., 2009). Similarly, variation in population responses to climate change are predicted across a species' range, as populations at the warmest range edge may be expected to have larger responses than populations in the middle of a species range (e.g., Beever et al., 2011). Thus, mammals within biogeographic regions of their range that are experiencing larger changes in climate may be predicted to show stronger responses to climate change.

Finally, which species do and do not respond to climate change may be a result of phylogenetic relatedness (Blomberg \& Garland, 2002; Blomberg et al., 2003; 
Ives \& Garland, 2010). If certain groups of mammals are consistently responding more than others due to a shared traits leading to climate change susceptibility, such a trend could lead to more persuasive arguments about species risk in areas of the world currently understudied for climate change response. In North America, the 73 species studied for responses belong to 11 orders and 16 families, representing about half of the worldwide mammal orders. The clade-based trends in climate change response can be assessed through estimates of phylogenetic signal in predictor variables and with comparisons between phylogenetically-corrected and uncorrected analyses (Ives \& Garland, 2010).

Given that some mammals are and some are not responding to current climate change, here we assess whether variability in species' traits, geographic location, and mammalian clade affiliation are consistently related to measured responses. We assess several mammal species' traits which may influence their interaction with a changing climate, including body size, activity times, burrowing behavior, and hibernation. We assess several biogeographic factors, including latitude of geographic range, location of the study population within the geographic range, and elevational range. Lastly, we assess the degree of response due to phylogenetic relatedness.

\section{Materials and methods}

\section{Mammal data}

Terrestrial mammal responses to anthropogenic climate change were located through standardized literature searches (ISI Web of Knowledge, Google Scholar, and Scirus) using various combinations of keywords (climate change, global warming, mammals) and those combinations also with locations specified (USA, Canada). In total we examined over 54000 citations of which about 1050 were relatively relevant. From these, we only included studies that empirically examined and statistically tested pre- and post- anthropogenic climate change resurvey data or long-term monitoring data across a time period of anthropogenic climatic change on native species based on the individual author's assessments of pertinent time intervals. We did not include studies that could not exclude anthropogenic habitat change as the main driver of change, those that did not have strong similarities in data collection methodology between the pre- and post-climate change periods, and those that only examined shifts in response to current climate and extended their results to predictions in the future (see Data S1 for more detail). Almost all of the included studies were from North America (NA: US, Canada, Greenland; Figure S1, Appendix S1; 73 species), whereas only a few studies met those criteria outside of NA (Appendix S2; 8 at species level). To be consistent about the degree of climate change experienced by the mammals, here we concentrated on the responses of NA mammals, although the results did not change with the inclusion of the 8 non-NA species' responses. A strong publication bias is not apparent in the NA data as nearly an equal number of populations and species are included that responded to climate change as those that did not respond or responded opposite to predictions (Figure S2a). This is not the case in non-NA data as only one study published a non-significant response (Poroshin et al., 2010; Appendix S2). Additionally, species' traits in response datasets are nearly identical (body size) or closely similar in distribution (activity times, latitudinal midpoints and maxima) to all NA terrestrial mammals, thus are unbiased in relationship to the analyzed predictor variables (Figure S2b-e). Data quality and statistical inferences still varied among included studies; therefore, we also analyzed a best data subset (Appendix S4) as well as two additional restricted datasets on extinction risk responses and contrasting contracting and expanding elevational ranges (see below).

Studies predicted seven climate change responses: local population extirpations, range contractions, range shifts, and directional change in abundance, phenology, body size, or genetic diversity. Responses were 'expected' if that was the predicted response specified in the particular study or according to climate change theory (e.g., upward elevational or poleward range shift), 'unexpected' if they were the opposite response than predicted (e.g., range expansion), and 'no change' if no response was detected. Local population extirpations were defined as complete loss of a species from a known locality or region. Range contractions were defined as an overall decrease in area of the latitudinal or elevational range of a species. Range shifts were defined as a latitudinal or elevational shift upward or downward of a species' range beyond historical limits without necessarily changing range size. At the latitudinal or regional level, many studies did not assess the entirety of a species' range but rather detected changes at local sites (e.g., Gleason \& Rode, 2009; Myers et al., 2009), therefore these are considered range shifts. Since range contraction and range shift can both be expected responses along elevational gradients (e.g., Moritz et al., 2008; Rowe et al., 2010; Beever et al., 2011) (and latitudinal gradients, although none are tested so far for mammals), we defined the 'expected' trend as contraction if the upper range limit abutted or was close to the mountain top, whereas the 'expected' trend for range shift was upslope if the upper limit was at a sufficiently low elevation to allow both range limits to shift upward without a change in range size. The 'unexpected' response to contraction was range expansion, with an increase in range size, and the 'unexpected' response to an upslope range shift was a downslope range shift without an increase in range size.

Population abundance changes were detected with increases or decreases in local abundance in comparison to values measured before detectable climate change, and predictions depended on the hypotheses presented in the study. Mammalian phenological changes assessed shifts in timing of reproductive events, hibernation, and other physiological events, and in all cases included here were expected to advance in their timing with increasing temperatures. Mammalian morphological changes associated with climate change were mostly concerned with body size shifts. Smaller body sizes were expected with increasing temperatures (e.g., Smith 
et al., 1998) or increases in body size were expected with a longer growing season (e.g., Ozgul et al., 2010), although some studies also discussed other morphological and genetic trends with climate change.

We examined all these responses as a whole for all datasets, including 147 population responses from 73 species (Appendix S1 and S3). We also analyzed a best data subset (80 populations of 49 species; Appendix S4), which included only those studies with greater than 5 years of sampling, least amount of potential anthropogenic habitat change, and with the strongest statistical inference-meaning the study showed (i) a strong, measurable relationship between climate change and the mammalian response variable; (ii) the sampling effort per method of assessing mammalian responses across the monitoring period or the historical and current periods was relatively consistent; and (iii) the climate change signal was shown to be stronger statistically than other explanatory variables. Because not all of the expected responses to climate change are potentially detrimental to the species, we also analyzed a subset of responses that are potentially linked to a higher extinction risk: local population extirpations, contracting ranges, and decreasing population sizes (66 populations of 35 species; Appendix S3). Lastly, we compared mammals that had contracted to those that had expanded their elevational ranges (36 populations of 19 species; Appendix S3).

Species' traits and biogeographic characteristics were assessed from the studies themselves and from the literature. Body sizes were from the MOM database (Smith et al., 2003) except Tamias minimus which was from the PanTHERIA database (Jones et al., 2009). Daily activity times (obligate diurnal, obligate nocturnal, and flexible [crepuscular, combination or flexibility of nocturnal, diurnal, crepuscular]) were from the PanTHERIA database (Jones et al., 2009), species accounts in the journal Mammalian Species, and checked against additional literature sources (Hall, 1981; Nowak, 1991; Armstrong et al., 2011). Burrowing, nesting behavior, hibernation, and torpor were compiled from various literature sources (Hall, 1981; Nowak, 1991; Matocq \& Murphy, 2007; Armstrong et al., 2011) and Mammalian Species accounts. Latitudinal range midpoints and maxima, and study locations within NA geographic ranges were calculated from the PanTHERIA database (Jones et al., 2009) but modified to only include distributions within NA (Hall, 1981; Matocq \& Murphy, 2007; IUCN, 2011). For contracting and expanding ranges, elevational range midpoint was an inaccurate reflection of overall range position due to the broad elevational ranges of most species. Therefore, a range was noted as (i) predominantly low elevation $(<$ midpoint of mountain) or all elevations vs.; (ii) predominantly high elevation (>midpoint of mountain). Because activity times may not be as wellknown among species that are difficult to see and monitor, the determination of flexible species might be under-represented. Therefore, we added a 'potentially flexible' category to the species if a literature source suggested that it may be active at other times or if a sister species or closely related species was well-known to be flexible in its activity times (Appendix S3 and S4). We then conducted comparison analyses to detect if this broader dataset influenced the results for the two datasets with sufficient numbers of potentially flexible species (all data, best data).

\section{Statistical analyses}

Analyses were conducted at the level of species to accommodate phylogenetic corrections (Blomberg \& Garland, 2002; Blomberg et al., 2003; Ives \& Garland, 2010) and avoid multiple population bias. Composite responses were used for species with multiple study populations. If one or more populations had detections of expected responses to climate change, then a positive response was noted for that species even if other populations detected no change. These composite responses changed between the complete dataset (Appendix S3) and the best subset (Appendix S4) for a few species with multiple studied populations of varying quality.

The climate change responses were analyzed as a binomial - either an expected response (1) or no response (0: unexpected or no response) per species. The unexpected responses were too few to be analyzed separately (eight species) and were almost all elevational expansions (Appendix S1; Figure S1). Thus, these were analyzed specifically in the comparison of contracting and expanding elevational ranges (Appendix S3). Only the elevational shifts were quantifiable as meters changed, but preliminary analyses were weak, mainly due to small sample sizes and restricted shift magnitudes. More quantified shift studies are needed before robust effect-size meta-analyses are possible. As noted in the appendices, for species trait and biogeographic variables, predictors were analyzed as continuous quantitative variables, two-state variables or three-state variables. For activity times (obligate diurnal, obligate nocturnal, and flexible), two dichotomies were strongest (flexible vs. obligate diurnal and nocturnal, and obligate diurnal vs. flexible and nocturnal) so both contrasts were included as two-state variables in the multivariate models.

In order to detect the best-fit models and the strongest predictor variables, we first conducted multivariate, nominal logistic regressions for all possible models using all combinations of predictor variables (Burnham \& Andersen, 2002). Second, we calculated AICc weights for each model and the model with the largest AICc weight was used as the best-fit model (Burnham \& Andersen, 2002). Third, to detect the influence of phylogenetic relatedness on each predictor variable and within the best-fit models, we ran both multivariate ordinary and phylogenetic logistic regressions (OLR, PLR) using the program PLogreg in MATLAB (Ives \& Garland, 2010). Phylogenies were from the mammal supertree (Bininda-Emonds et al., 2007) pruned to the taxa included in each dataset. PLogreg also estimated the phylogenetic signal with confidence intervals for each variable or model, which allows determination of response differences among mammalian clades. Phylogenetic signal in PLogreg varies from no signal ( -4$)$ to strong signal (>0) (Ives \& Garland, 2010). Body size was log-transformed, all predictor variables were standardized to a mean of zero and a variance of one, and all bootstrapped confidence intervals calculated with 2000 simulations following Ives \& Garland (2010). PLogreg was not used in the original assessment of the best-fit models, 
because a maximum of only three predictors variables was possible in a single model with our sample sizes to produce reliable results (e.g., model convergence, completed simulations) and PLogreg cannot calculate AICc weights (Ives \& Garland, 2010). Lastly, due to the small sample sizes for contracting and expanding elevational ranges $(n=19)$, only 3 variables were needed for a complete fit model $\left(r^{2}=1.0\right)$, thus AICc weights were calculated only for models of three variables or less. For more details on analyses and models see the Data S1.

\section{Results}

Only $52 \%$ of mammal populations responded as expected to recent climate change. In contrast, $7 \%$ responded opposite to expectations and $41 \%$ had no detectable response (Figure S1; Appendix S1). The majority of mammalian populations were assessed for range contraction, range shifts, and abundance changes (82\%) with fewer studies examining other responses (local extirpations, phenology, morphological or genetic changes: 18\%). The best-fit models for the various datasets all included two species' traits, body size and activity times, whereas latitudinal and elevational range were only included in a few models (Tables S1 and S2). In contrast, burrowing, nesting, hibernation, heterothermy, and study location within geographic range were not included in any of the best-fit models, and were rarely significant in single variable models (Tables S1 and S2). For the dataset of all species and responses (73 species), the best-fit model included only body size and flexible activity times $\left(r^{2}=0.22 ; X^{2}=22.09, P<0.0001\right)$. The best-fit models for the best subset of species ( 49 species) and the extinction risk dataset (35 species) included body size, flexible activity times, latitudinal range $\left(r^{2}=0.50 ; X^{2}=32.60, P<0.0001 ; r^{2}=0.71 ; X^{2}=29.54\right.$, $P<0.0001$, respectively). Lastly, the best-fit model for the species contracting and expanding their elevational ranges (19 species) included body size, diurnal activity times, elevational range $\left(r^{2}=1.0 ; \quad X^{2}=23.70, \quad P<\right.$ $0.0001)$. The statistical significance of the best-fit models and strongest predictor variables were identical with and without phylogenetic correction (Tables S1 and S2).

Body size was the strongest single predictor of climate change response across all datasets (Fig. 1). Expected responses to climate change increased with body size with and without phylogenetic corrections in all best-fit models (Table S2; OLR P-values: 0.005$<0.0001$; PLR P-values: 0.045-<0.0001). For the mammals studied, body sizes ranged from $2.5 \mathrm{~g}$ to $388 \mathrm{~kg}$, and mammals at about $100 \mathrm{~g}$ shifted to predominantly responding to climate change. The largest mammals, e.g., elk and polar bear, were 27 times more likely to respond to climate change than were the smallest mammals, e.g., shrews and mice.

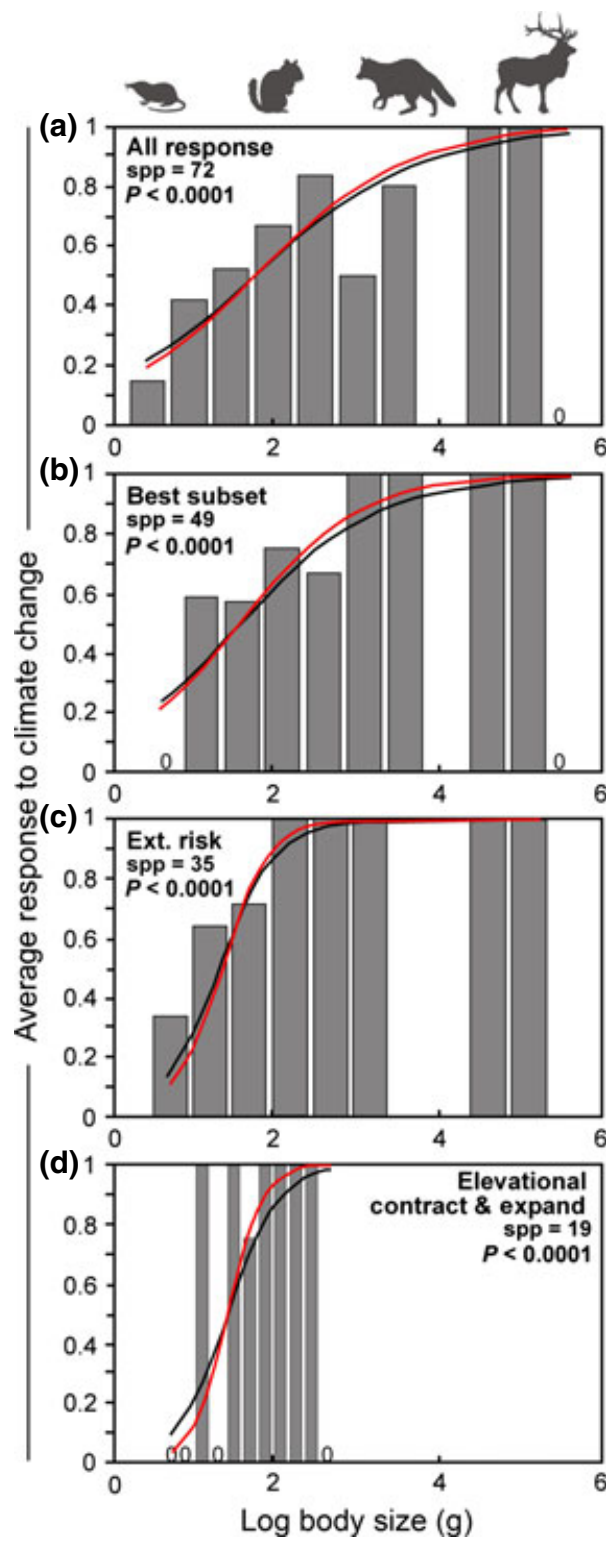

Fig. 1 Responses to anthropogenic climate change increase with mammalian body size shown using ordinary (red line) and phylogenetic logistic regression (black line). (a) All species and all responses to climate change. (b) Best data subset and all responses to climate change. (c) Only those species tested for responses potentially linked to extinction risk (local population extirpation, range contraction, decreasing population size). For (a-c), climate change responses: expected response $=1$, and no response or unexpected response $=0$. (d) Larger species are more likely to contract their elevational ranges (1), whereas smaller mammals are more likely to expand their elevational ranges (0). $P$-values are from single variable, ordinary logistic regression models. Zeros in the figures indicate where certain body size ranges have an average response to climate change of zero (i.e. no response).

Species with flexible activity times had significantly lower response rates to climate change than obligate diurnal or nocturnal species (Fig. 2a; multivariate OLR 
$P$-values: 0.012-0.001; multivariate PLR P-values: 0.0180.004). Diurnal species contracted their elevational ranges, whereas flexible and nocturnal species were more likely to expand their ranges (Fig. 2b; multivariate $P$ - values: $\mathrm{OLR}<0.0001$; PLR $=0.020$ ). Analyses were robust to including more species as potentially flexible (single and multivariate models still significant; Tables S1 and S2). Activity was not just a function of body size as the two variables were not significantly associated $(r=0.012, P=0.349)$, and both variables were included and significant in all four best-fit, multivariate models.

The biogeographic variables were not as strongly or consistently related to mammalian responses to climate change as were species' traits (Tables S1 and S2). Only latitudinal range measured as either midpoint or maximum had some consistent support among datasets (Fig. 3). Climate change responses increased with increasing latitude of species' ranges in most single variable models (except all responses), and in best-fit models for the best data and extinction risk data (Table

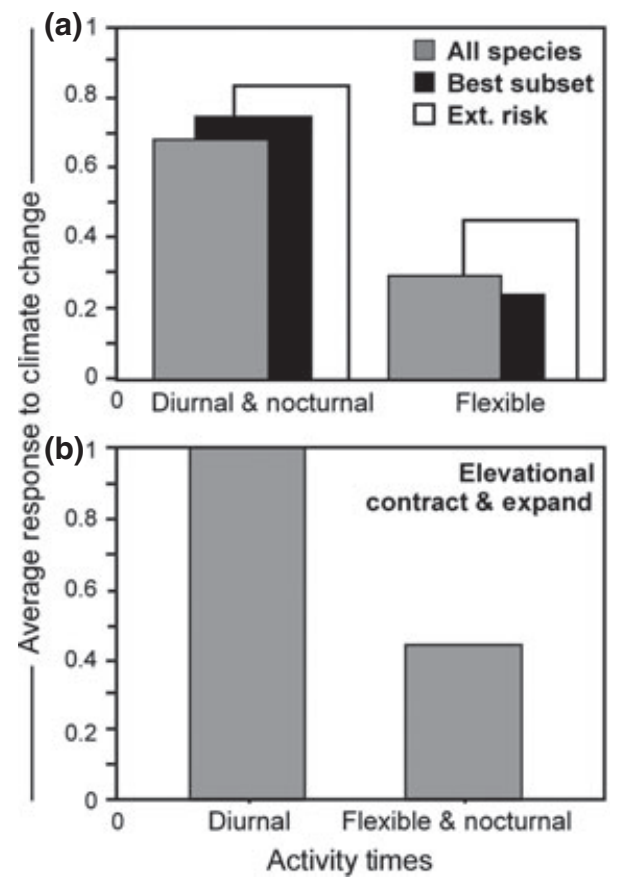

Fig. 2 Responses to anthropogenic climate change decrease with flexible activity times using ordinary and phylogenetic logistic regressions. (a) For all species (grey; $n=72, P=0.001$ ), the best subset (black; $n=49, P<0.0001$ ) and extinction risk responses (white; $n=35, P=0.016$ ). Climate change responses: expected responses $=1$, and no response or unexpected response $=0$. (b) The species contracting their elevational ranges (1) are all diurnal, whereas species that expanded their elevational ranges $(0)$ were either nocturnal or flexible in their activity times $(n=19, P<0.0001)$. $P$-values are from single variable, ordinary logistic regression models.
S2; best: OLR $P<0.0001$ and 0.002; PLR $P<0.0001$ and 0.021 , respectively). For changing elevational ranges (Fig. 4), species with higher elevation ranges predominantly contracted their ranges, whereas species with lower elevation ranges expanded their ranges (OLR $P=0.012$, PLR $P=0.033$ ).

Estimates of phylogenetic signal using PLogreg were low among all datasets and variables: all bootstrapped confidence intervals included -4 , which corresponds to no detectable phylogenetic signal (Tables S1 and S2; Ives \& Garland, 2010). Nonetheless, all point estimates of phylogenetic signal were larger than -4 and denote a small level of phylogenetic relatedness influencing the distribution of climate change responses. Thus, variability in responses existed within and across clades, and particular mammalian clades were not associated strongly with climate change responses. Regardless, both the ordinary and phylogenetic logistic regressions consistently supported the same variables as strongly linked to the differences in climate change responses, albeit the phylogenetically-corrected $P$-values are generally slightly larger (Tables S1 and S2).

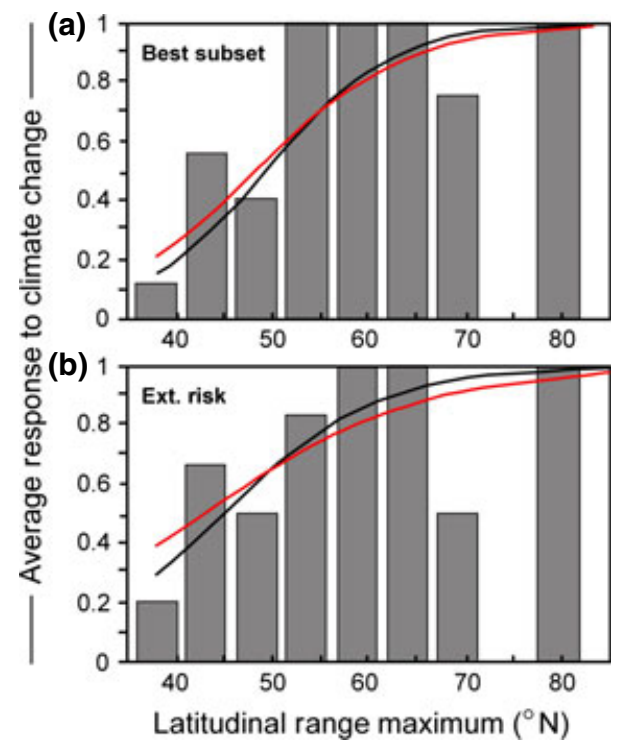

Fig. 3 Responses to anthropogenic climate change increase with the latitude of a species' range shown for latitudinal maximum (midpoints are nearly identical; not shown) using ordinary (red line) and phylogenetic logistic regression (black line). (a) Best data and all responses to climate change $(n=49$, $P<0.0001$ ); (b) only those species tested for responses potentially linked to extinction risk (local population extirpation, range contraction, decreasing population size): $(n=35$, $P=0.005)$. Climate change responses: expected response $=1$; no response or unexpected response $=0 . P$-values are from single variable, ordinary logistic regression models. 

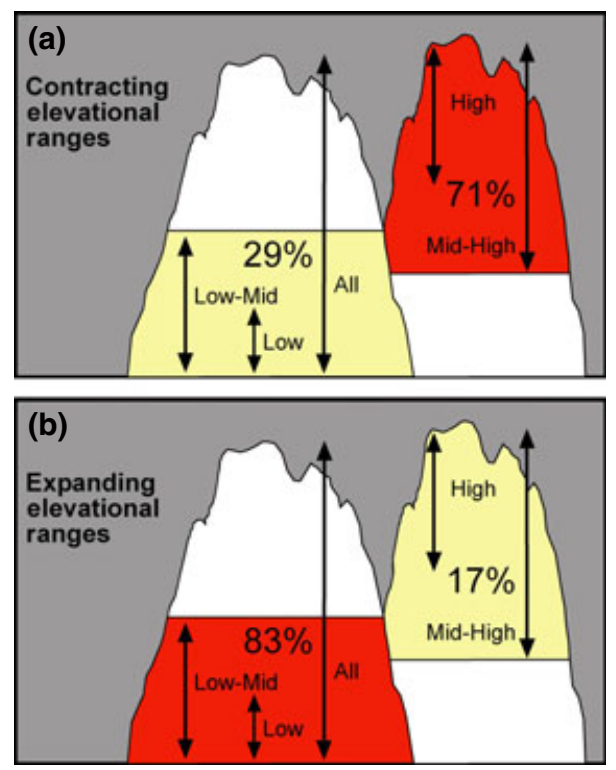

Fig. 4 Mammal species that have contracted their elevational range were predominately detected at high elevation (a), whereas species that have expanded their elevational range were predominately detected at low elevation or across both low and high elevations (b). Higher response rates are shown in red and lower response rates in yellow; and arrowed lines represent the elevational ranges of the groups included in each analysis. This relationship between contracting and expanding elevational ranges was significant in single variable, ordinary logistic regression models $(P=0.031)$.

\section{Discussion}

Mammalian studies examining current climate change responses clearly demonstrate that not all mammals are responding or responding similarly to climate change. Some mammals are responding negatively with local population extirpations, range contractions, and decreasing population sizes, for example polar bears, pika, and Shadow chipmunks (Moritz et al., 2008; Gleason \& Rode, 2009; Beever et al., 2011). Other mammals are responding positively by increasing ranges, population size, or growth rates, for example several shrews and Yellow-bellied marmots (Moritz et al., 2008; Ozgul et al., 2010; Rowe et al., 2010). And a substantial portion of mammals have not responded at all, nearly half of those studied so far. Other studies documenting multiple species' responses to climate change in other taxonomic groups including insects and plants have detected responders and non-responders (Grabherr et al., 1994; Lenoir et al., 2008; Chen et al., 2009; Angert et al., 2011). Identification of biological reasons underlying this variation in response to climate change will aid in our ability to develop robust and targeted conservation plans and make more informed management decisions for those species most at risk.
Variability in climate change response may be due to species' traits that influence how they interact with their climatic environment (sensitivity) and how pronounced the climate change is in a particular site (exposure) (e.g., Huey et al., 2012). For ectotherm vertebrates, researchers have suggested that behavioral thermoregulation, acclimation, and adaptation as well as other life history characteristics could have cascading influences on species' responses (e.g., Buckley, 2008; Huey et al., 2012). For endotherms there have been fewer suggestions of important species' traits due to the assumption that they are not as directly tied to ambient conditions as ectotherms. And previous attempts to detect species' traits linked to the magnitude of climate change responses in birds and mammals have not been successful (Angert et al., 2011). Paleontologists have suggested that certain mammal traits like body size, burrowing, and nocturnality helped them survive extreme climatic events (K-T extinction; Robertson et al., 2004). In support of the sheltering hypotheses, we robustly detected two mammalian traits that influence an endotherm's response based on variability in a species' exposure and sensitivity to prevailing climatic conditions - body size and activity times.

Body size and activity times were consistently and strongly related to the variability in mammalian responses to climate change. Expected responses increased with body size for all types of responses and just for responses indicating potential extinction risk (i.e. local extirpations, range contractions, and population declines). Pleistocene mega-faunal extinction included predominantly large-bodied mammals (Barnosky et al., 2004). The largest Pleistocene range shifts were detected among the large bodied mammals, including the larger rodents (Lyons, 2003). In contrast, the smallest bodied mammals are consistently represented in the North American fossil record across the past 80 Myr (Alroy, 1998), and they survived the Cretaceous-Tertiary boundary, while larger, non-avian dinosaurs became extinct (Robertson et al., 2004; Lloyd et al., 2008). During the Pleistocene, small mammals did not show an increased extinction rate or as many large range shifts as larger mammals (Lyons, 2003; Barnosky et al., 2004; Blois et al., 2010). This body size trend may indicate climatic interactions not previously considered in mammalian responses to current climate change. Smaller mammals may experience climate differently than larger mammals due to differential availability of microhabitats and thus their microclimates near and under the soil and vegetation where both temperature and humidity is moderated (Kay, 1977; Bulova, 2002; Feldhamer et al., 2007; Porter \& Kearney, 2009; Scherrer \& Körner, 2011; Suggitt et al., 2011; Huey et al., 2012; Scheffers et al., 2013). Larger 
mammals may have less flexibility in the climate and temperatures they encounter above the understory vegetation due to their necessary exposure to ambient air temperature, humidity and daily-seasonal variability. This may suggest that climate grain size scales with body size (sensu Ritchie, 2010), and that the larger grain of climate encompasses more climate change than does the smaller grain of climate available to smaller mammals. Additionally, mammal body size has repercussions on many life history characteristics from metabolic and reproductive rates to population sizes and longevity that may not be as directly related to climate, but have predictable relationships with rarity and general extinction risk (e.g., Cardillo et al., 2005; Feldhamer et al., 2007; Moritz et al., 2008; Liow et al., 2009). Large mammals may be more mobile and therefore better able to expand their ranges to track changes and encounter refuges (e.g., Angert et al., 2011; Schloss et al., 2012). However, not only may this be confounded by habitat loss or fragmentation, but our result that large mammals are contracting their ranges and decreasing in abundance more than small mammals indicates a stronger negative impact of climate change than a simple increased mobility response.

The variability of activity times among mammals from nocturnal or diurnal to behavioral flexibility also had strong repercussions on the heterogeneity in climate change responses. The species with flexibility in activity times-ability to switch between some combination of nocturnal, diurnal, and crepuscular activitywere the least likely to respond or respond negatively to climate change. In fact, many of these species were those that responded positively by increasing their elevational ranges or abundances. Whereas those that were obligately active at a particular time of day responded at much higher rates and with more negative responses. In particular, on mountains it appears that those species obligately diurnal are most at risk, and this may be due to the cold adapted species at high elevation possessing a lower physiological tolerance for the warmest daily temperatures (e.g., pika: Beever et al., 2011 and references therein). This activity trait result, although surprising, pinpoints an important mammalian trait difference that has cascading importance on the ability of a species to behaviorally influence their experienced climate. Flexible species can select a particular set of climatic conditions that may be significantly different from the ambient conditions from which a nocturnal or diurnal species cannot escape. This species' trait may have been similarly important in paleo-mammals, but is not a trait easily identified with fossil evidence.

Temperatures are more moderate and less variable near the soil and under vegetative ground cover during both summer and winter, and are also more stable in underground environments (Kay, 1977; Bulova, 2002; Feldhamer et al., 2007), thus burrowing and nesting mammals may have less exposure to changing climate. But these traits were not strongly linked to current climate change responses. This may be because this is a basal trait within most mammals. Most Mesozoic mammals are thought to be burrowers and nesters, and this basal trait dominates in extant mammals (Hall, 1981; Nowak, 1991). Among the mammals studied for climate change responses, fewer than five species did not burrow or nest (e.g., artiodactyls, some large carnivores), while the rest burrowed, built nests, or both. Burrowing and nesting traits may be less important for climate responses within mammals than among vertebrate clades. Hibernation and daily torpor allow some mammals to survive extreme temperatures and low food resource conditions by metabolic manipulations (Feldhamer et al., 2007; Armstrong et al., 2011). Despite the strong association with climatic adaptation, hibernation and torpor traits were not linked to the variability in responses to current climate change, suggesting that species with or without heterothermy capabilities are not particularly more or less sensitive to climate change, at least in North America.

Responses to climate change are predicted to be concentrated geographically (Grabherr et al., 1994; Walther et al., 2002; Parmesan \& Yohe, 2003; Root et al., 2003; Hickling et al., 2006), although biogeographic factors were shown to be secondary to species' traits in this study. Higher latitude species were more likely to respond to climate change, but only in the best data subset and extinction risk responses. High elevation species were strongly associated with range contraction for montane mammals. Despite the stronger trends for body size and activity, high latitude and elevation still appear to be highly susceptible environments.

Other reasons for no detectable climate change response may be due to additional anthropogenic causes, for example survey data are incomplete or sparse, or anthropogenic habitat modifications are stronger (Walther et al., 2002; Parmesan \& Yohe, 2003; Root et al., 2003; Lenoir et al., 2008; Rowe et al., 2010). Additionally, time lags, non-climatically determined range limit or abundance trends, strong phenotypic plasticity, and larger tolerance limits than currently expressed may lead to not detecting a climate change response (Walther et al., 2002; Parmesan \& Yohe, 2003; Root et al., 2003; Lenoir et al., 2008; Rowe et al., 2010). In our analyses, we control for data quality and responses to other anthropogenic habitat changes through a comparison of all response data to a best data subset. We cannot control or test for time lags or larger tolerance limits than currently exhibited until more data become available. 
In summary, our results suggest that large-bodied, obligatory diurnal or nocturnal mammals are rapidly responding to current climate change and many of these responses indicate higher extinction risks. Most of these mammals are the charismatic fauna of North America: bighorn sheep, pika, and polar bears. Those least likely to respond or that are expanding their ranges are those small, flexible, unseen mammals: the shrews and mice living in the soil and under the vegetation. Microclimate availability and behavioral choices on activity times, even for two species on the same plot of land, therefore, have cascading implications for the climate experienced, and dictate the necessity of responding by tracking a climate niche or escaping from change (Porter \& Kearney, 2009; Kearney et al., 2010; Scherrer \& Körner, 2011; Suggitt et al., 2011; Huey et al., 2012; Scheffers et al., 2013). More research documenting the climate change responses in mammals throughout the world is urgently needed, and these results give a framework for assessing mammal traits and the pertinent climate change scale most likely associated with differential responses to climate change. Since most predictive models of climate change risk treat all mammals as equally likely to respond (e.g., Moritz et al., 2008; McCain \& Colwell, 2011), including body size and activity times will improve predictions with evolutionary and biological realism to increase utility for conservation planning.

\section{Acknowledgements}

This work was supported by the US National Science Foundation (McCain: DEB 0949601). We thank all researchers whose data were used in this study, and Robert Anderson, Robert Colwell, Daniel Doak, Jaelyn Eberle, Norman Slade, and anonymous reviewers for feedback on manuscript drafts.

\section{Author contributions}

C.M.M. formulated the ideas, conducted the analyses, and wrote the manuscript. S.R.B.K. conducted the literature search, helped collect the papers and data, and provided feedback on ideas, analyses, and manuscript revisions.

\section{References}

Adamik P, Král M (2008) Climate-and resource-driven long-term changes in dormice populations negatively affect hole-nesting songbirds. Journal of Zoology, 275, 209215.

Alroy J (1998) Cope's rule and the dynamics of body mass evolution in North American fossil mammals. Science, 280, 731-734.

Angert AL, Crozier LG, Rissler LJ, Gilman SE, Tewksbury JJ, Chunco AJ (2011) Do species' traits predict recent shifts at expanding range edges? Ecology Letters, 14, 677-689.

Aragón P, Rodríguez MA, Olalla-Tárraga MA, Lobo JM (2010) Predicted impact of climate change on threatened terrestrial vertebrates in central Spain highlights differences between endotherms and ectotherms. Animal Conservation, 13, 363 373.

Armstrong DM, Fitzgerald JP, Meaney CA(2011) Mammals of Colorado. University Press of Colorado, Denver, $\mathrm{CO}$.

Barnosky AD, Koch PL, Feranec RS, Wing SL, Shabel AB (2004) Assessing the causes of late Pleistocene extinctions on the continents. Science, 306, 70-75.

Beever EA, Ray C, Wilkening JL, Brussard PF, Mote PW (2011) Contemporary climate change alters the pace and drivers of extinction. Global Change Biology, 17, 2054 2070.

Bininda-Emonds ORP, Cardillo M, Jones KE et al. (2007) The delayed rise of modern mammals. Nature, 444, 93-96.

Blois JL, Mcguire JL, Hadly EA (2010) Small mammal diversity loss in response to late-Pleistocene climatic change. Nature, 465, 771-774.

Blomberg SP, Garland T (2002) Tempo and mode in evolution: phylogenetic inertia, adaptation and comparative methods. Journal of Evolutionary Biology, 15, 899-910.

Blomberg SP, Garland T, Ives AR (2003) Testing for phylogenetic signal in comparative data: behavioral traits are more labile. Evolution, 57, 717-745.

Buckley LB (2008) Linking traits to energetics and population dynamics to predict lizard ranges in changing environments. American Naturalist, 171, E1-E19.

Bulova SJ (2002) How temperature, humidity, and burrow selection affect evaporative water loss in desert tortoises. Journal of Thermal Biology, 27, 175-189.

Burnham KP, Andersen DR (2002) Model selection and multi-model inference: a practical information-theoretic approach. Springer-Verlag, Heidelberg.

Cardillo M, Mace GM, Jones KE et al. (2005) Multiple causes of high extinction risk in large mammal species. Science, 309, 1239-1241.

Chen IC, Shiu HJ, Benedick S et al. (2009) Elevation increases in moth assemblages over 42 years on a tropical mountain. Proceedings of the National Academy of Sciences of the United States of America, 106, 1479-1483.

Duffy PB, Tebaldi C (2012) Increasing prevalence of extreme summer temperatures in the U.S. Climatic Change, 111, 487-495.

Feldhamer GA, Drickamer LC, Vessey SH, Merritt JF, Krajewski C (2007) Mammalogy: adaptation, diversity, ecology Baltimore. The Johns Hopkins University Press, Maryland.

Gingerich PD (2006) Environment and evolution through the Paleocene-Eocene thermal maximum. Trends in Ecology \& Evolution, 21, 246-253.

Gleason JS, Rode KD (2009) Polar bear distribution and habitat association reflect long-term changes in fall sea ice conditions in the Alaskan Beaufort Sea. Arctic, 62 405-417.

Grabherr G, Gottfried M, Pauli H (1994) Climate effects on mountain plants. Nature, 369,448 .

Hall ER (1981) Mammals of North America. John Wiley \& Sons, New York.

Hickling R, Roy DB, Hill JK, Fox R, Thomas CD (2006) The distributions of a wide range of taxonomic groups are expanding polewards. Global Change Biology, 12, 450-455.

Huey RB, Kearney MR, Krockenberger A, JaM Holtum, Jess M, Williams SE (2012) Predicting organismal vulnerability to climate warming: roles of behaviour, physiology and adaptation. Philosophical Transactions of the Royal Society B: Biological Sciences, 367, 1665-1679.

IUCN (2011) IUCN Red List of Threatened Species. Version 2011.2. http:// www. iucnredlist.org (accessed Feb-Aug 2012).

Ives AR, Garland T (2010) Phylogenetic logistic regression for binary dependent variables. Systematic Biology, 59, 9-26.

Jones KE, Bielby J, Cardillo M et al. (2009) PanTHERIA: a species-level database of life history, ecology, and geography of extant and recently extinct mammals. Ecology, 90, 2648-2648.

Kay FR (1977) Environmental physiology of the banner-tailed kangaroo rats II. Influences of the burrow environment on metabolism and water loss. Comparative Biochemistry and Physiology Part A: Physiology, 57, 471-477.

Kearney MR, Wintle BA, Porter WP (2010) Correlative and mechanistic models of species distribution provide congruent forecasts under climate change. Conservation Letters, 3, 203-213.

Lawler JJ, Shafer SL, White D, Kareiva P, Maurer EP, Blaustein AR, Bartlein PJ (2009) Projected climate-induced faunal change in the Western Hemisphere. Ecology, 90, 588-597.

Lenoir J, Gegout JC, Marquet PA, De Ruffray P, Brisse H (2008) A significant upward shift in plant species optimum elevation during the 20th century. Science, 320, 1768-1771.

Liow LH, Fortelius M, Lintulaakso K, Mannila H, Stenseth NC (2009) Lower extinction risk in sleep-or-hide mammals. American Naturalist, 173, 264-272.

Lloyd GT, Davis KE, Pisani D et al. (2008) Dinosaurs and the cretaceous terrestrial revolution. Proceedings of the Royal Society B: Biological Sciences, 275, 2483-2490. 
Lundy M, Montgomery I, Russ J (2010) Climate change-linked range expansion of Nathusius' pipistrelle bat, Pipistrellus nathusii (Keyserling \& Blasius, 1839). Journal of Biogeography, 37, 2232-2242.

Lyons SK (2003) A quantitative assessment of the range shifts of Pleistocene mammals. Journal of Mammalogy, 84, 385-402.

Matocq MD, Murphy PJ (2007) Fine-scale phenotypic change across a species transition zone in the genus Neotoma: disentangling independent evolution from phylogenetic history. Evolution, 61, 2544-2557.

McCain CM, Colwell RK (2011) Assessing the threat to montane biodiversity from discordant shifts in temperature and precipitation in a changing climate. Ecology Letters, 14, 1236-1245.

Moritz C, Patton JL, Conroy CJ, Parra JL, White GC, Beissinger SR (2008) Impact of a century of climate change on small-mammal communities in Yosemite National Park, USA. Science, 322, 261-264.

Moyes K, Nussey DH, Clements MN et al. (2011) Advancing breeding phenology in response to environmental change in a wild red deer population. Global Change Biology, 17, 2455-2469.

Myers P, Lundrigan BL, Hoffman SMG, Haraminac AP, Seto SH (2009) Climateinduced changes in the small mammal communities of the Northern Great Lakes Region. Global Change Biology, 15, 1434-1454.

Nowak RM (1991) Walker's Mammals of the World. The John Hopkins University Press, Baltimore \& London.

Ozgul A, Childs DZ, Oli MK et al. (2010) Coupled dynamics of body mass and population growth in response to environmental change. Nature, 466, 482-485.

Parmesan C (2006) Ecological and evolutionary responses to recent climate change. Annual Review of Ecology Evolution and Systematics, 37, 637-669.

Parmesan C, Yohe G (2003) A globally coherent fingerprint of climate change impacts across natural systems. Nature, 421, 37-42.

Poroshin EA, Polly PD, Wojcik JM (2010) Climate and morphological change on decadal scales: Multiannual variation in the common shrew Sorex araneus in northeast Russia. Acta Theriologica, 55, 193-202.

Porter WP, Kearney M (2009) Size, shape and the thermal niche of endotherms. Proceedings of the National Academy of Sciences, 106, 19666-19672.

Post E, Forchhammer MC (2008) Climate change reduces reproductive success of an Arctic herbivore through trophic mismatch. Philosophical Transactions of the Royal Society B: Biological Sciences, 363, 2367-2373.

Ritchie ME (2010) Scale, Heterogeneity, and the Structure and Diversity of Ecological Communities. Princeton University Press, Princeton, New Jersey.

Robertson DS, Mckenna MC, Toon OB, Hope S, Lillegraven JA (2004) Survival in the first hours of the cenozoic. Geological Society of America Bulletin, 116, 760-768.

Root TL, Price JT, Hall KR, Schneider SH, Rosenzweig C, Pounds JA (2003) Fingerprints of global warming on wild animals and plants. Nature, 421, 57-60.

Rowe RJ, Finarelli JA, Rickart EA (2010) Range dynamics of small mammals along an elevational gradient over an 80-year interval. Global Change Biology, 16, 2930-2943.

Scheffers BR, Brunner RM, Ramirez SD, Shoo LP, Diesmos A, Williams SE(2013) Thermal buffering of microhabitats is a critical factor mediating warming vulnerability of frogs in the Philippine biodiversity hotspot. Biotropica, 45, 628-635.
Scherrer D, Körner C (2011) Topographically controlled thermal-habitat differentiation buffers alpine plant diversity against climate warming. Journal of Biogeography, 38, 406-416.

Schloss CA, Nuñez TA, Lawler JJ (2012) Dispersal will limit ability of mammals to track climate change in the Western Hemisphere. Proceedings of the National Academy of Sciences, 109, 8606-8611.

Smith FA, Browning H, Shepherd UL (1998) The influence of climate change on the body mass of woodrats Neotoma in an arid region of New Mexico, USA. Ecography, 21, 140-148.

Smith FA, Lyons SK, Ernest SKM et al. (2003) Body mass of late quaternary mammals. Ecology, 84, 3403

Suggitt AJ, Gillingham PK, Hill JK, Huntley B, Kunin WE, Roy DB, Thomas CD (2011) Habitat microclimates drive fine-scale variation in extreme temperatures. Oikos, 120, 1-8.

Trenberth KE, Jones PD, Ambenje P et al. (2007) Observations: surface and atmospheric climate change. In: Climate Change 2007: The Physical Science Basis. Contribution of Working Group I to the Fourth Assessment Report of the Intergovernmental Pane on Climate Change. (eds Solomon S, Qin D, Manning M, Chen Z, Marquis M, Averyt KB, Tignor M, Miller HL), pp. 235-336. Cambridge University Press, Cambridge, United Kingdom and New York, NY, USA.

USGCRP (2009) Global Climate Change Impacts in the United States. (eds Karl TR Melillo JM, Peterson TC). United States Global Change Research Program. Cambridge University Press, New York, NY.

Walther GR, Post E, Convey P et al. (2002) Ecological responses to recent climate change. Nature, 416, 389-395

\section{Supporting Information}

Additional Supporting Information may be found in the online version of this article:

Data S1. Methods.

Appendix S1. North America (NA) database and literature cited.

Appendix S2. Outside NA data.

Appendix S3. All NA species dataset.

Appendix S4. Best NA subset dataset.

Figure S1. Response categories.

Figure S2. Sample data comparison.

Table S1. Ordinary and phylogenetic logistic regression statistics.

Table S2. Best-fit AIC multivariate models. 